\title{
BLIND EXTRACTION OF DIGITAL WATERMARKING ALGORITHM FOR COLOR IMAGES
}

\author{
Xuecheng Gong, Wanggen Li and Wang Jing \\ School of Computer and Information, Anhui Normal University, Anhui Wuhu, China
}

\begin{abstract}
Digital watermark technology hides copyright information in digital images, effectively protecting the copyright of digital images. At present, the color image digital watermarking algorithm still has defects such as the inability to balance robustness, invisibility and the weak anti-attack ability. Aiming at the above problems, this paper studies the digital watermarking method based on discrete wavelet transform and discrete cosine transform. Then this paper proposes a color image blind digital watermarking algorithm based on QR code. First, convert the color image from RGB space to YCbCr space, extract the Y component and perform the second-level discrete wavelet transform. secondly, block the LL2 subband and perform the discrete cosine transform. finally, use the embedding method to convert the watermark information after the Arnold transform embedded in the block. The experimental results show that the PSNR of the color image embedded with the QR code is 56.7159 without being attacked. After being attacked, its PSNR and NC values are respectively $30 \mathrm{~dB}$ and 0.95 or more, which proves that the algorithm has good robustness and can achieve watermarking blind extraction.
\end{abstract}

\section{KEYWORDS}

QR Code; Color Image; Arnold Transform; Discrete Wavelet Transform; Discrete Wavelet Transform

\section{INTRODUCTION}

With the development of the Internet, various digital works have appeared on the Internet. At the same time, with the rapid development of information digitization, there has also been a problem that copyright is not easy to protect[1]. Due to the ease of dissemination and copying of digital images, all kinds of piracy have emerged one after another and the copyright protection of digital images has become increasingly serious. Regarding the copyright protection and copyright authentication of digital media products, the traditional copyright protection method is information encryption, which processes digital information through a certain mathematical method, so that it cannot be correctly identified without knowing the encryption method and key. Information encryption protects copyright to a certain extent, but traditional information encryption methods will disrupt encrypted information and cause damage to digital information[2].

As a method of solving copyright protection problems, digital watermarking is a technology that embeds designated digital identification information into digital products transmitted over the network to protect product copyrights and does not affect the use of digital products[3]. The content of the watermark can be the name of the work, creation time or corporate identity, etc. The watermark content can prove the copyright owner and can also be used as proof of copyright protection. According to different embedding methods, it can be divided into spatial domain DOI:10.5121/ijma.2021.13202 
The International Journal of Multimedia \& Its Applications (IJMA) Vol.13, No. 1/2, April 2021

watermark and transform domain watermark. At present, embedding the digital watermark into the carrier image transform domain can better improve the robustness and security of the watermark. Quick response code (quick response code) is abbreviated as QR code, which is characterized by storing a large amount of information, low cost, small size, fast display of information and error correction function[4]. Arnold transform has periodicity and is widely used in image scrambling. Using its characteristics to transform the position of QR code information can improve the security of watermarking[5].

\section{RELATED WORK}

In order to study the digital watermarking algorithm in depth. First, introduce the discrete wavelet transform and discrete cosine transform used in the digital watermarking algorithm in this paper. Then introduce the Arnold watermark encryption method and finally introduce the watermark performance evaluation.

QR code can store not only text information, but also image information. In actual use, due to the tilt of the shooting, it will be difficult to read and sometimes the QR code will be distorted. The QR code corrects the image and detects data errors through position detection graphics and positioning graphics to ensure the integrity of the data. When the code is distorted, the data can still be recovered through the error correction code. Therefore, using the QR code as a watermark cannot only improve the robustness of the watermark, but also store more copyright information.

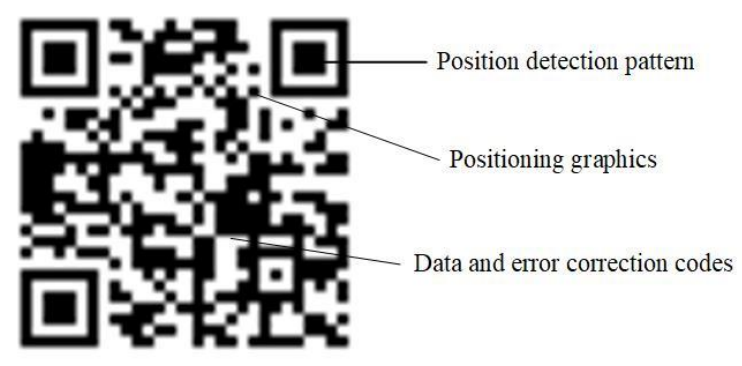

Figure 1. QR code structure

He et al[6]. proposed a color image watermarking algorithm based on discrete wavelet transform, discrete cosine transform and singular value decomposition (DWT-DCT-SVD). First, convert the carrier image from the RGB color space to the YUV color space; then, perform a layer of discrete wavelet transform on the brightness component $\mathrm{Y}$, use discrete cosine transform to decompose the low frequency and its blocks and perform singular value decomposition on each block; Finally, embed the watermark into the carrier image. However, there is a problem with the DWTDCT-SCD method. A non-blind watermark image is needed to extract the watermark in the experiment and the watermark non-QR code is used in the experiment.

$\mathrm{Xu}$ Jiangfeng et al[7]. proposed a digital watermarking scheme combining QR code, chaotic system and DWT-DCT. Carry out DWT operation on the carrier image and perform $4 \times 4$ block and DCT operation on the low-frequency subbands, embed the QR code watermark through the chaotic system into the carrier image. The experimental results show that after the Gaussian noise attack, the PSNR value and the NC value are low and the experiment uses gray-scale images, which is less practical. To solve the above problems, this paper proposes a color image blind digital watermarking scheme based on QR code. This scheme selects a color image as the carrier image, converts the RGB color space to the $\mathrm{YCbCr}$ color space, embeds the watermark into the luminance component $\mathrm{Y}$ and uses the Arnold transformation to encrypt the QR code, which not 
only improves the security and robustness of the watermark, but also Increased the amount of watermark information.

Poonam et al[8]. proposed a robust digital watermarking method that uses DWT and SVD technology to insert the watermark into the singular value of the carrier image frequency domain subband. The experimental results show that the technology has significant imperceptibility. Improved, with remarkable robustness when subjected to different attacks.

Sunesh et al[9]. proposed a blind extraction of digital watermark technology using discrete cosine transform. This is achieved by embedding the watermark into the DCT coefficients of the images at two different locations. Embedding the same watermark bit in two different locations helps to maintain security and robustness. Experiments have proved that the proposed watermarking algorithm has good perceptual ability and low computational complexity.

Sinhal et al[10]. proposed an effective blind extraction and easy-to-detect watermarking scheme for color images. This solution can detect tampering or forgery in the image and effectively restore the tampered part. It can be effectively executed against different types of tampering attacks. In addition, the proposed solution provides self-recovery of the tampered area of the image and effectively recovers the highly tampered image with acceptable parameters.

Pan et al[11]. proposed a watermarking technology based on the SMS algorithm, which uses improved discrete wavelet transform and singular value decomposition to hide the watermark in the QR code and adjusts the embedding strength factor through the SMS algorithm to modify the strength of the watermark embedded in different frequency domains. The experimental result has a higher PSNR value and the QR code can still be decoded by a general decoder. The proposed method is feasible and effective.

\subsection{Discrete Wavelet Transform}

In digital image processing, it is necessary to discretize the continuous wavelet and its wavelet transform. The discretized wavelet and its corresponding wavelet transform are called discrete wavelet transform[12]. Discrete wavelet transform is a spatio-temporal scale analysis method of information analysis theory and signal. It has multiple scales in space and frequency domain and can continuously decompose images from low resolution to high resolution[13,14]. In addition, the DWT algorithm has a wide range of applications in the digital watermarking neighborhood, and many innovative and efficient joint algorithms related to DWT have been proposed.

In this paper, the RGB color space of the color carrier image is transferred to the YUV color space and the Y component is DWT to obtain the low-frequency LL in the horizontal and vertical directions, the low-frequency in the horizontal direction and the high-frequency LH in the vertical direction, the high-frequency in the horizontal direction and the low-frequency HL in the vertical direction. And high-frequency components $\mathrm{HH}$ in the horizontal and vertical directions. DWT is performed on the LL subband again to obtain the low frequency component LL2. The low-frequency components represent image features, the high-frequency components represent the edges and details of the image. Embedding the watermark in the low frequency component LL2 can improve the robustness. 
The International Journal of Multimedia \& Its Applications (IJMA) Vol.13, No. 1/2, April 2021

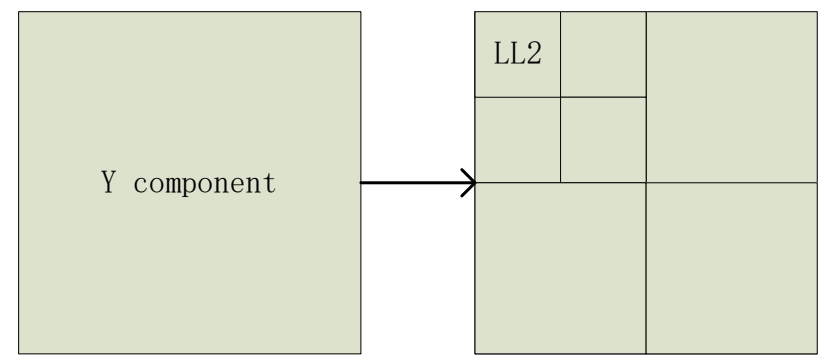

Figure 2. decomposition diagram

\subsection{Discrete Cosine Transform}

The principle of Discrete Cosine Transform is to discretize even-numbered terms in the expansion of Discrete Fourier Transform. Discrete Cosine Transform can convert spatial domain signals to frequency domain signals and has good decorrelation performance[15]. The twodimensional DCT transform can be obtained by two one-dimensional transforms and the DCT transform is a reversible transform, often used in signal processing and image compression[16].

The digital image is processed by DCT transformation to obtain low frequency, intermediate frequency and high frequency[17]. The low frequency part is the part in the image related data set. The high frequency part is watermarked, and the watermark information is easily lost when attacked. The intermediate frequency part is the best position for watermarking. The important information of the image after the discrete cosine transform is concentrated on the middle and low frequency coefficients and the position is the upper left corner of the DCT matrix, which has the ability to resist attacks. After being attacked, the embedded watermark information can still be extracted. In this paper, the LL2 subband is divided into blocks, the blocks are subjected to DCT transformation and then the medium and low frequency coefficients are selected for watermark embedding, the DCT inverse transformation completes the image reconstruction., As shown in the formula (1) and (2), f(i,j) is the original signal, $F(u, v)$ is the coefficient after DCT transformation, $\mathrm{N}$ is the number of points of the original signal, $\mathrm{c}(\mathrm{u})$ and $\mathrm{c}(\mathrm{v})$ can be considered as the compensation coefficient and the DCT transformation matrix can be made into an orthogonal matrix.

$$
\begin{gathered}
F(u, v)=c(u) c(v) \sum_{i=0}^{N-1} \sum_{j=0}^{N-1} f(i, j) \cos \left[\frac{(i+0.5) \pi}{N} u\right] \cos \left[\frac{(j+0.5) \pi}{N} v\right](1) \\
c(u)=\left\{\begin{array}{cc}
\sqrt{\frac{1}{N}} & u=0 \\
\sqrt{\frac{2}{N}} & u \neq 0
\end{array}\right.
\end{gathered}
$$

\subsection{Arnold Transformation}

Arnold transformation is also called cat face transformation, which transforms a meaningful image into a meaningless image by scrambling the image. The watermark image contains important information[18]. The Arnold transform is used to scramble the image to achieve information encryption. At the same time, the Arnold transform is periodic and the number of scrambling can be used as the watermark key to further enhance its security. The periodicity of Arnold transformation refers to the continuous transformation of the original image and the 
The International Journal of Multimedia \& Its Applications (IJMA) Vol.13, No. 1/2, April 2021

original image is obtained after $\mathrm{T}$ times. The transformation period $\mathrm{T}$ is related to the size of the image $\mathrm{M} \times \mathrm{N}[19]$. The Arnold transformation is defined as follows:

$$
\left[\begin{array}{l}
\mathrm{x}^{*} \\
\mathrm{y}^{*}
\end{array}\right]=\left[\begin{array}{ll}
1 & 1 \\
1 & 2
\end{array}\right]\left[\begin{array}{l}
\mathrm{x} \\
\mathrm{y}
\end{array}\right](\bmod \mathrm{N})(3)
$$

Among them, $(\mathrm{x}, \mathrm{y})$ represents the pixel position before image scrambling, $\left(\mathrm{x}^{*}, \mathrm{y}^{*}\right)$ represents the image pixel location after scrambling and $\mathrm{N}$ represents the order of the image matrix.

\section{EMBEDDING AND EXTRACTION OF WATERMARK}

This article uses QR code as the watermark map, which adds more information and improves the security of the watermark. The use of color images as carrier images is more widely used.

\subsection{Embedding of watermark}

a) Convert the color carrier image from RGB color space to $\mathrm{YCbCr}$ color space according to the algorithm flow and extract the brightness component $\mathrm{Y}$.

b) Perform a two-level DWT transformation on the luminance component to obtain the low frequency subband LL2, then implement $2 \times 2$ block division on the LL2 subband to obtain a block matrix.

c) Perform DCT transformation on each block (dct=dct(LL2)) to obtain the transformed DCT matrix. Then extract the first value in the matrix from the DCT transformed block to form a new matrix $F$.

d) Use Arnold transform algorithm to scramble the original watermark image $\mathrm{W}$ to get the scrambled watermark $\mathrm{W}^{*}$.

e) Embed the watermark $\mathrm{W}^{*}$ into the matrix $\mathrm{F}$ using equations (4) and (5) to obtain the matrix $\mathrm{F}^{*}$. Then replace each value of the matrix $\mathrm{F}^{*}$ with the first value of each block in turn, and perform inverse DCT transformation on each block to obtain LL2*.

$$
\begin{gathered}
\lambda_{1}^{*}=\left\{\begin{array}{c}
\lambda_{1}-T+3 a / 4 \quad T \geq a / 4 \\
\lambda_{1}-T-a / 4 \quad \text { other }
\end{array} W^{*}(i, j)=0\right. \\
\lambda_{1}^{*}=\left\{\begin{array}{cc}
\lambda_{1}-T+5 a / 4 & T \geq 3 a / 4 \\
\lambda_{1}-T+a / 4 & \text { other }
\end{array} W^{*}(i, j)=1(5)\right.
\end{gathered}
$$

Among them, $T=\lambda \_1$ moda, $a$ is the embedding strength. Used to control the invisibility and robustness of embedded watermarks. $\lambda 1$ represents each value in each block matrix. $\lambda \_1^{\wedge *}$ Represents the embedded value.

f) Implement the second-level inverse DWT transformation on the obtained in the previous step to obtain the component. The brightness component of the embedded QR code is converted from the YCbCrcolor space to the RGB color space to obtain the color carrier image embedded in the QR code. Figure 3 is a flowchart of watermark embedding. 
The International Journal of Multimedia \& Its Applications (IJMA) Vol.13, No. 1/2, April 2021

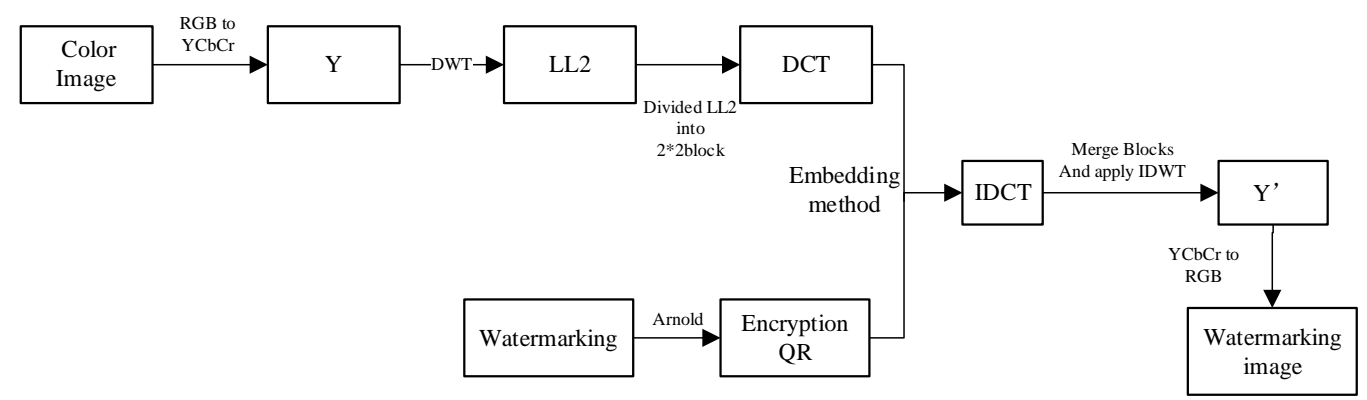

Figure 3. Watermarking embedding flow chart.

\subsection{Extract Watermark}

a) First, convert the color carrier image embedded with QR code from RGB color space to $\mathrm{YCbCr}$ color space and extract the brightness component $\mathrm{Y}^{*}$.

b) Perform two-level DWT transformation on the luminance component to obtain the lowfrequency subband. Then perform $2 \times 2$ block on the subband.

c) Perform DCT transformation on each block to obtain the transformed DCT matrix. $\mathrm{dct}=\mathrm{dct}(\mathrm{LL} 2 *)$. Then extract the first value in the matrix from the block after DCT transformation to form a matrix $\mathrm{F}^{*}$.

d) The extraction of watermark information is the reverse process of watermark embedding. The watermark is extracted by equations (6).

$$
W(i, j)=\left\{\begin{array}{cc}
1 & T>a / 2 \\
0 & \text { other }
\end{array}(6)\right.
$$

e) According to the obtained $\mathrm{W}^{*}$ in the previous step. Then perform Arnold transformation on it. Finally the watermark W is extracted. Figure 4 is a flow chart of watermark extraction.

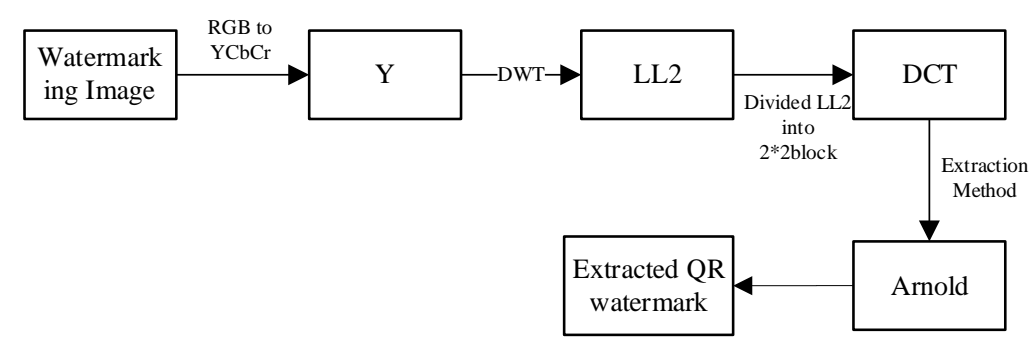

Figure 4. Watermarking extraction flow chart.

\section{The EXPERIMENTAL RESUlts}

The experimental environment is: Intel Core i5-4210M CPU; $2.60 \mathrm{GHz}$ frequency; Windows 10 64-bit operating system; Matlab2018a software. Select the color carrier image with $512 \times 512$ pixels, the $64 \times 64 \mathrm{QR}$ code is the watermark and the QR code carries the information. Figure 5(a) is a color carrier image, and Figure 5(b) is a QR code watermark image. 
The International Journal of Multimedia \& Its Applications (IJMA) Vol.13, No. 1/2, April 2021

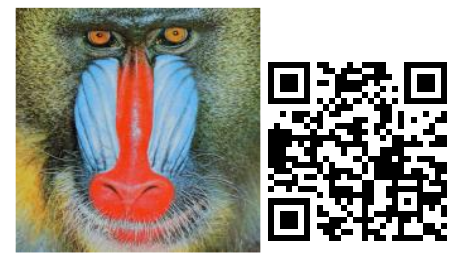

a)Color Image $\quad$ (b)QR Code

Figure 5. Experiment Pictures.

\subsection{Watermark Evaluation standard}

Whether the performance of digital watermarking algorithm reaches the standard of research and application is mainly judged by two aspects: one is the invisibility of watermark, which is evaluated by subjective evaluation of human eyes or quantitative calculation of objective methods. The second is the robustness of watermark, that is the watermark resistance to attack, which means whether the watermark image can extract effective watermark information after being attacked. The performance evaluation criteria and indexes of the two watermarking algorithms are described below.

Watermark invisibility refers to the high similarity between the original image and the watermarked image. One detection method is that the human eye cannot detect the watermark information hidden in the image and does not affect the use of the original image, so the invisibility of the watermark algorithm is better. Another detection method is to detect invisibility by using performance evaluation indicators, signal-to-noise ratio (SNR), peak signal-to-noise ratio (PSNR) and mean square error (MSE).

Experiments usually use peak signal-to-noise ratio (PSNR) to measure the difference between the QR code-embedded image and the unembedded original image. The greater the PSNR, the higher the recognition of the image embedded in the QR code with the original image. The definition of PSNR is:

$$
\mathrm{PSNR}=10 \log _{10} \frac{255^{2} \mathrm{M} \times \mathrm{N}}{\sum_{\mathrm{x}=0}^{\mathrm{M}-1} \sum_{\mathrm{y}=0}^{\mathrm{N}-1}\left(\mathrm{I}^{*}(\mathrm{x}, \mathrm{y})-\mathrm{I}(\mathrm{x}, \mathrm{y})\right)^{2}}(7)
$$

Among them, represents a watermarked color carrier image and I represents a color carrier image. When the PSNR value is greater, the color carrier image is closer to the watermarked color carrier image. The embedded watermark effect is better. PSNR $>30 \mathrm{~dB}$ usually means that the watermark is invisible and the image quality is better.

Robustness means that the watermarking algorithm can resist common attacks such as compression, clipping, noise, and clipping. Generally speaking, if the digital watermark is highly robust, the digital watermark has strong anti-attack ability. In the experimental part of this article, the normalized correlation coefficient (NC) is used as an objective index to evaluate the attacked watermark. The robustness of the watermark is detected by comparing the original watermark information and the watermark extracted from the carrier image. NC represents the similarity between the original watermark and the extracted watermark. The NC value ranges from 0 to 1 . The closer the $\mathrm{NC}$ value is to 1 , the higher the similarity between the original watermark and the extracted watermark. The definition of $\mathrm{NC}$ is: 
The International Journal of Multimedia \& Its Applications (IJMA) Vol.13, No. 1/2, April 2021

$$
\mathrm{NC}=\frac{\sum_{\mathrm{x}=0}^{\mathrm{M}-1} \sum_{\mathrm{y}=0}^{\mathrm{N}-1} \mathrm{~W}(\mathrm{x}, \mathrm{y}) \mathrm{W}^{*}(\mathrm{x}, \mathrm{y})}{\sqrt{\sum_{\mathrm{x}=0}^{\mathrm{M}-1} \sum_{\mathrm{y}=0}^{\mathrm{N}-1} \mathrm{~W}(\mathrm{x}, \mathrm{y})^{2} \sum_{\mathrm{x}=0}^{\mathrm{M}-1} \sum_{\mathrm{y}=0}^{\mathrm{N}-1} \mathrm{~W}^{*}(\mathrm{x}, \mathrm{y})^{2}}}(8)
$$

Among them, W represents the original watermark image. represents the extracted watermark image.

\subsection{Analysis of Results}

In order to test the robustness of the algorithm, the experiment uses JPEG compression, noise filtering, cropping attack, rotation attack and median filtering to attack watermarked images.

a) JPEG compression.

Table 1. JPEG Compression.

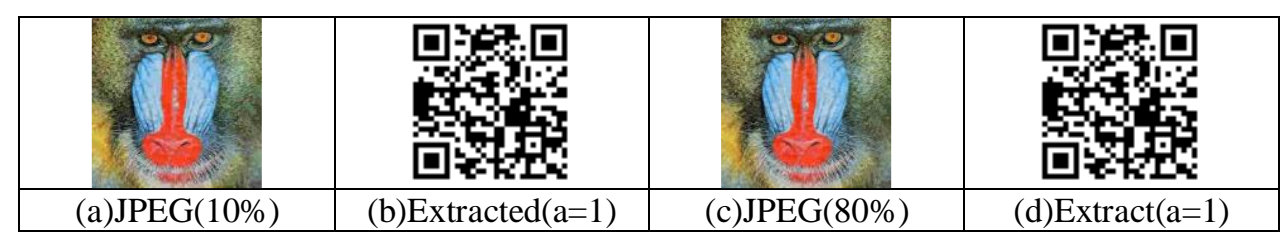

In the experiment, the JPEG compression attack was performed on the image. Table 1(a)-(d) are the results of the JPEG compression attack under different factors. It can be measured that the extracted QR code can be recognized by the machine.

According to the data in Table 2, when the JPEG compression factor is $10 \%$, the PSNR value is 48.9158 and the $\mathrm{NC}$ value is 1 . At this time, the QR code can still be identified, indicating that the algorithm in this paper is robust against JPEG compression attacks and can ensure the integrity of the watermark information.

Table 2. JPEG Compression attack data.

\begin{tabular}{|c|c|c|c|c|c|c|}
\hline \multirow{2}{*}{ Methods } & \multicolumn{7}{|c|}{ Compression Ratio } \\
\cline { 2 - 7 } & $\mathbf{1 0 \%}$ & $\mathbf{2 0 \%}$ & $\mathbf{3 0 \%}$ & $\mathbf{4 0 \%}$ & $\mathbf{6 0 \%}$ & $\mathbf{8 0 \%}$ \\
\hline PSNR/dB & 48.9158 & 50.0294 & 50.6425 & 51.0356 & 51.6767 & 52.5922 \\
NC & 1 & 1 & 1 & 1 & 1 & 1 \\
\hline
\end{tabular}

b) Noise attack. The experiment uses salt and pepper noise and Gaussian noise attacks, as shown in Table 3.

Table 3. Noise attack.

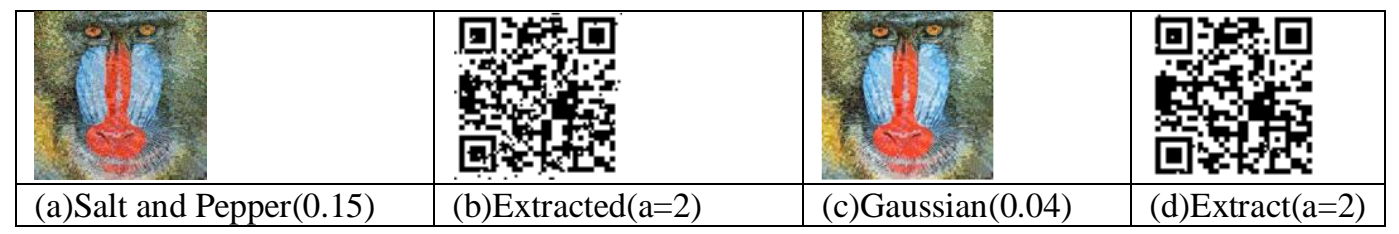


The International Journal of Multimedia \& Its Applications (IJMA) Vol.13, No. 1/2, April 2021

Table 4. Noise attack data.

\begin{tabular}{|l|l|l|l|l|l|l|l|}
\hline \multirow{2}{*}{ Method } & \multicolumn{9}{|l|}{ Salt and Pepper Noise } & \multicolumn{3}{l|}{ Gaussian Noise } \\
\cline { 2 - 9 } & $\mathbf{0 . 0 1}$ & $\mathbf{0 . 0 5}$ & $\mathbf{0 . 1 5}$ & $\mathbf{0 . 0 1}$ & $\mathbf{0 . 0 2}$ & $\mathbf{0 . 0 3}$ & $\mathbf{0 . 0 4}$ \\
\hline PSNR/dB & 45.3121 & 41.7544 & 37.9263 & 43.0325 & 42.9243 & 42.7836 & 42.5694 \\
\hline NC & 1 & 1 & 09687 & 1 & 0.9997 & 09995 & 0.9962 \\
\hline
\end{tabular}

It can be seen from Table 4 that the PSNR value of the color carrier image embedded with the QR code is attacked by salt and pepper noise and Gaussian noise respectively. The PSNR value is also above $30 \mathrm{~dB}$. It indicating that the attacked carrier image shows strong robustness and can resist noise attack. From the perspective of the NC value, the extracted watermark maintains a high consistency with the original watermark and the extracted watermark can be identified.

c) Cropping attack. It can be seen from the different cropping areas in Table 6 that the QR code is embedded in the frequency domain of the carrier image. The cropping of different areas can still maintain a high NC value, the PSNR value can also be maintained above $30 \mathrm{~dB}$ and the QR code can be Identified. Therefore, this algorithm has good resistance to shearing attacks.

Table 5. Cropping attack.

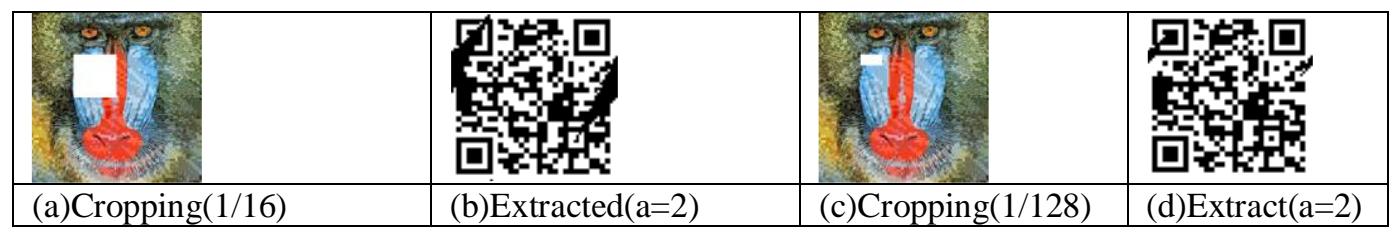

Table 6. Cropping attack data.

\begin{tabular}{|l|l|l|l|l|l|l|}
\hline \multirow{2}{*}{ Method } & \multicolumn{5}{l}{ CroppingRatio } & \multicolumn{1}{l|}{$l \mid$} \\
\cline { 2 - 7 } & $\mathbf{1 / 8}$ & $\mathbf{1 / 1 6}$ & $\mathbf{1 / 3 2}$ & $\mathbf{1 / 6 4}$ & $\mathbf{1 / 1 2 8}$ & $\mathbf{1 / 2 5 6}$ \\
\hline PSNR/dB & 41.3916 & 41.3724 & 43.0706 & 45.3883 & 45.8662 & 46.0592 \\
\hline NC & 0.9600 & 0.9589 & 0.9782 & 0.9868 & 0.9928 & 0.9951 \\
\hline
\end{tabular}

d) Rotation attack and Median filtering. It can be concluded from Table 7 that the algorithm can resist rotation attacks and median filtering. The PSNR values are all above $30 \mathrm{~dB}$ and the $\mathrm{NC}$ values remain above 0.9 . It shows that the algorithm has good robustness.

Table 7. Rotation attack and Median filtering data.

\begin{tabular}{|l|l|l|l|}
\hline \multirow{2}{*}{ Method } & \multicolumn{2}{l|}{ Rotation Angle } & Median Filtering \\
\cline { 2 - 4 } & $\mathbf{5}^{\circ}$ & $\mathbf{1 0}$ & {$[\mathbf{3} \times \mathbf{3}]$} \\
\hline PSNR/dB & 42.4715 & 40.4322 & 45.2474 \\
\hline NC & 0.9693 & 0.9558 & 0.9991 \\
\hline
\end{tabular}

e) Comparison results. It can be seen from Table 8 that in the median filter attack, the algorithm in this paper is better than the non-blind QR code watermarking algorithm in the literature [7]. In this paper, the $\mathrm{NC}$ values of Gaussian noise and salt and pepper noise are both higher than 0.9. When the JPEG compression factor is $30 \%$, the NC value reaches 1 , 
The International Journal of Multimedia \& Its Applications (IJMA) Vol.13, No. 1/2, April 2021

which is higher than the $\mathrm{NC}$ value in the literature [7]. It can be concluded that the algorithm in this paper is more robust than the algorithm in [7].

Table 8. Compare the results of different experiments.

\begin{tabular}{|l|l|l|}
\hline Attacks & $\begin{array}{l}\text { NC(Proposed } \\
\text { Method) }\end{array}$ & $\begin{array}{l}\text { NC(Reference } \\
[7])\end{array}$ \\
\hline JPEG Compression 30\% & 1 & 0.9875 \\
\hline Cropping 1/16 & 0.9589 & - \\
\hline Gaussian Noise 0.04 & 0.9962 & 0.9300 \\
\hline Salt and Pepper Noise 0.05 & 1 & 0.9849 \\
\hline Median Filtering [3×3] & 0.9991 & 0.9873 \\
\hline
\end{tabular}

As shown in Figure 6, use the following images to conduct non-attack and attack experiments. Perform watermark extraction and NC value calculation on watermarked images to check whether the scheme in this chapter can be applied to a wide range of digital images.

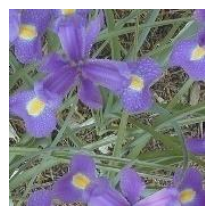

flower

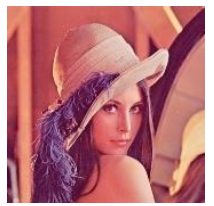

Lena

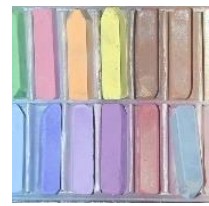

middle

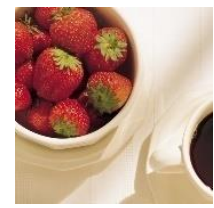

strawberries

Figure 6. Experiment Pictures.

As shown in the table 9, when the image is not attacked, the NC value of the extracted watermark information is equal to 1; after the attack, the NC value of most of the extracted watermark information is in the range of [0.9-1]. It is proved that the method proposed in this chapter can resist common attacks and the extracted watermark information is similar and consistent with the original watermark. The algorithm has good performance and the watermark is robust.

Table 9. Compare the results of different experiments.

\begin{tabular}{|l|l|l|l|l|l|l|}
\hline Methods & unattack & $\begin{array}{l}\text { JPEGComperess } \\
\mathbf{3 0 \%}\end{array}$ & $\begin{array}{l}\text { Median } \\
\text { Filtering } \\
{[\mathbf{3} \times \mathbf{3}]}\end{array}$ & $\begin{array}{l}\text { Gaussian } \\
\text { Noise } \\
\mathbf{0 . 0 1}\end{array}$ & $\begin{array}{l}\text { Rotation } \\
\mathbf{5}^{\circ}\end{array}$ & $\begin{array}{l}\text { Cropping } \\
\mathbf{1} / \mathbf{6 4}\end{array}$ \\
\hline flower & 1 & 1 & 1 & 0.9938 & 0.9731 & 0.9859 \\
\hline Lena & 1 & 1 & 1 & 0.9899 & 0.9732 & 0.9861 \\
\hline middle & 1 & 0.9998 & 0.9965 & 0.9938 & 0.9750 & 0.9876 \\
\hline strawberries & 1 & 0.9982 & 0.9978 & 0.9786 & 0.9735 & 0.9874 \\
\hline
\end{tabular}

In order to verify that the algorithm is universal, this article adds experimental images to verify the reliability of the experiment. The experimental data is shown in the table. The normalized correlation coefficients of the extracted watermarks are calculated for each image in the table without attack and under attack. From the calculated data, it can be known that the algorithm in this paper has good robustness to various attacks, it can resist external attacks and can be used for color image digital watermarking. 
The International Journal of Multimedia \& Its Applications (IJMA) Vol.13, No. 1/2, April 2021

\section{Conclusions}

In this paper, color images are used as carrier images and QR codes are used as watermark information, which increases the amount of watermark information carried and also improves the watermark's anti-attack ability. The implementation of Arnold transformation on QR code and implementation of DWT, DCT and block operations on the carrier image effectively strengthen the robustness of the watermark. By embedding watermarks on multiple color images, the algorithm still shows good anti-attack ability, indicating that the algorithm can be used for color images. The attack experiment data also shows that the algorithm in this paper is resistant to JPEG compression, clipping attack, Gaussian noise, salt and pepper noise, median filter and rotation attack, the PSNR value is above $30 \mathrm{~dB}$. The algorithm proposed in this paper also has shortcomings. In a rotation attack, when the rotation angle exceeds $12^{\circ}$, the extracted QR code will not be recognized and information cannot be obtained through the device. In view of the above problems, the algorithm needs to be continuously improved.

\section{ACKNOWLEDGEMENTS}

This work is supported by the Fund Project: Project of the Leading Talents in Colleges and Universities of Anhui Province(gxfxZD2016014).The authors would like to thank my teacher for his guidance and the anonymous reviewers for providing valuable comments on the manuscript.

\section{REFERENCES}

[1] P. Rasti\& S. Samiei, M, et al,(2016) "Robust non-blind color video watermarking using QR decomposition and entropy analysis," J. Vis. Commun. Image Represent., vol. 38, no. 5,pp. 838-847.

[2] J. Wang \& Z. Du, (2019)“A method of processing color image watermarking based on the Haar wavelet," J. Vis. Commun. Image Represent., vol. 64, pp. 102627.

[3] S. Kushlev\& R. P. Mironov, (2020) "Analysis for Watermark in Medical Image using Watermarking with Wavelet Transform and DCT," 2020 55th Int. Sci. Conf. Information, Commun. Energy Syst. Technol. ICEST 2020 - Proc., pp. 185-188.

[4] Bai TaoTao\& Liu Zhen, et al, (2014) "Contourlet domain digital watermarking algorithm based on QR code,"Optoelectronics'Laser,vol. 25, no. 4, pp. 769-776.

[5] Li Guohe\& Chen Chen, et al, (2018) "Digital watermark insertion and extraction method for QR code, "Computer Engineering and Applications, vol. 55, no. 10, PP. 103-107, 114.

[6] Y. He \& Y. Hu, (2018) "A Proposed Digital Image Watermarking Based on DWT-DCT-SVD," Proc. 2018 2nd IEEE Adv. Inf. Manag. Commun. Electron. Autom. Control Conf. pp. 1214-1218.

[7] Xu Jiangfeng\& Zhang Shouqiang, (2018) "DWT-DCT digital watermarking algorithm based on QR code," Application Research of Computers, vol. 35, no. 5, pp. 1540-1544.

[8] Poonam \& S. M. Arora, (2018) "A DWT-SVD based Robust Digital Watermarking for Digital Images," Procedia Comput. Sci., vol. 132, pp. 1441-1448.

[9] Sunesh\&R.Rama Kishore, (2020) "A Novel and Efficient Blind Image Watermarking in Transform Domain," Procedia Comput. Sci., vol. 167, no. 2019, pp. 1505-1514.

[10] R. Sinhal\& I. A. Ansari, et al, (2020) Ahn, "Blind Image Watermarking for Localization and Restoration of Color Images," IEEE Access, vol. 8, pp. 200157-200169.

[11] J. S. Pan \& X. X. Sun, et al, (2020) "Digital watermarking with improved SMS applied for QR code," Eng. Appl. Artif. Intell., vol. 97, no. November 2020, pp. 104049.

[12] Yuan Zihan\& Liu Decheng, et al, (2020) "New image blind watermarking method based on twodimensional discrete cosine transform," Optik (Stuttg), vol. 204, no. February 2020, pp. 164152.

[13] Zhu Jianzhong\& Yao Zhiqiang, (2014). "Color image blind watermarking algorithm based on DWTSVD and Turbo code," Journal of Jilin University: Science Edition, vol. 52, no. 4 pp. 773-778.

[14] Y. A. Mekarsari\& D. R. I. M. Setiadi, et al, (2018) "Non-blind RGB image watermarking technique using 2-level discrete wavelet transform and singular value decomposition," 2018 Int. Conf. Inf. Commun. Technol, vol. 2018-Janua, pp. 623-627. 
The International Journal of Multimedia \& Its Applications (IJMA) Vol.13, No. 1/2, April 2021

[15] M. Ali \& C. W. Ahn, et al, (2014) "A robust image watermarking technique using SVD and differential evolution in DCT domain," Optik (Stuttg), vol. 125, no. 1, pp. 428-434.

[16] D. O. Munoz-Ramirez \& V. Ponomaryov, et al, (2018) "A robust watermarking scheme to JPEG compression for embedding a color watermark into digital images," Proc. 2018 IEEE 9th Int. Conf. Dependable Syst. Serv. Technol, pp. 619-624.

[17] D. O. Muoz Ramirez \& V. Ponomaryov, et al, (2019) "Embedding a Color Watermark into DC coefficients of DCT from Digital Images," IEEE Lat. Am. Trans, vol. 17, no. 8, pp. 1326-1334.

[18] Wu Fengbo\& Wang Feng, (2016) "Wavelet transform digital image watermarking algorithm based on HVS," Applied Optics, vol. 35, no. 2, pp. 254-259.

[19] S. Saadi\& A. Merrad, (2019) "Novel secured scheme for blind audio/speech norm-space watermarking by Arnold algorithm," Signal Processing, vol. 154, pp. 74-86.

\section{AUTHORS}

Xuecheng Gong born in 1994, male, who studies at the Anhui Normal University, researching image processing.

Wanggen Li born in 1973, male, professor at the Anhui Normal University. His current research interests include biological computing and intelligent computing.

Wang Jing born in 1995, male, who studies at the Anhui Normal University, researching image processing and intelligent computing.
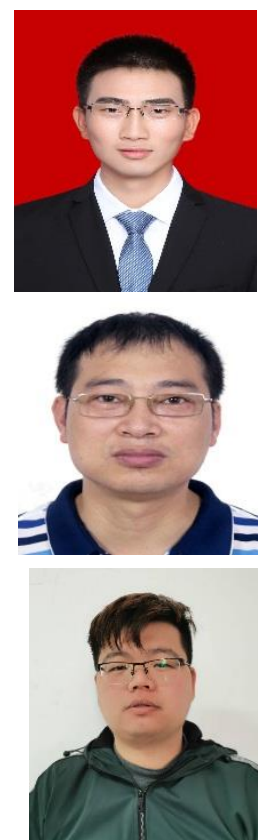